\title{
Highly Contiguous Genome Resource of Colletotrichum fructicola Generated Using Long-Read Sequencing
}

\author{
Xiaofei Liang, ${ }^{1}$ Mengyu Cao, ${ }^{1}$ Sen Li, ${ }^{1}$ Yuanyuan Kong, ${ }^{1}$ Jeffrey A. Rollins, ${ }^{2}$ Rong Zhang, ${ }^{1, \dagger}$ and \\ Guangyu Sun ${ }^{1, \dagger}$ \\ ${ }^{1}$ State Key Laboratory of Crop Stress Biology in Arid Areas and College of Plant Protection, Northwest \\ A\&F University, Yangling, 712100, Shaanxi Province, China \\ ${ }^{2}$ Department of Plant Pathology, University of Florida, Gainesville, FL, U.S.A.
}

\begin{abstract}
Colletotrichum fructicola is a plant-pathogenic fungus with a broad host range. It causes significant losses to important crops, including apple, pear, strawberry, and other Rosaceae and non-Rosaceae species. To date, two short read-based $C$. fructicola genomes are publicly available, but both are fragmented. In this study, we re-sequenced the genome of $C$. fructicola using nanopore long-read technology and refined the assembly with Hi-C map data. The resulting high-quality assembly is an important resource for further comparative and experimental studies with $C$. fructicola.
\end{abstract}

\section{Genome Announcement}

Colletotrichum fructicola Prihast., L. Cai \& K.D. Hyde was first identified from coffee (Coffea arabica) berries in 2009 (Prihastuti et al. 2009). It has since been reported from a wide range of plant species, such as tobacco, orchids, cassava, citrus, and tea-oil trees (Bragança et al. 2016; Hu et al. 2019; Li et al. 2016; Silva-Cabral et al. 2019; Wang et al. 2016). C. fructicola damages both foliar and fruit tissues and routinely impairs the commercial production of apple, strawberry, and pear. In addition to causing foliar and fruit diseases, C. fructicola can also associate with plants as an endophyte (Prihastuti et al. 2009). Such diverse plant associations and distinct plant-interaction lifestyles indicate that this broad hostrange fungus may comprise forms with distinct host preferences. Relevant to this hypothesis, isolates derived from diseased apple leaf and apple fruit infect in tissue-specific manners (Rockenbach et al. 2016). Genomic comparisons would be an important approach to revealing factors governing such pathogenic specificity variation. A high-quality reference genome is pivotal to conducting these analyses. To date, two $C$. fructicola genomes (Nara gc5, isolated from strawberry in Japan, and 1104-7, isolated from an apple leaf-spot lesion in China) are publicly available (Gan et al. 2013; Liang et al. 2018a). However, both genomes were generated with Illumina sequencing technology and are, thus, highly fragmented. Combining long-read sequencing with $\mathrm{Hi}-\mathrm{C}$ assisted scaffolding has been a powerful approach for generating chromosome-scale scaffolds (Ghurye et al. 2017). In this study, we resequenced isolate1104-7 with a long-read approach and further refined the assembly using $\mathrm{Hi}-\mathrm{C}$ mapping data.

Young mycelia of 1104-7 (deposited in the Agricultural Culture Collection of China, ACCC39328) was used as the source material for genome sequencing. Mycelia was harvested from a 200-ml 2-day old potato dextrose broth (PDB) shake culture inoculated with

\footnotetext{
${ }^{\dagger}$ Corresponding authors: R. Zhang; rongzh@nwsuaf.edu.cn and G. Sun; sgy@ nwsuaf.edu.cn
}

The author(s) declare no conflict of interest.

Accepted for publication 12 March 2020.

(C) 2020 The American Phytopathological Society

\section{Funding}

This work was supported by the National Key Research and Development Program of China (2016YFD0201132), National Natural Science Foundation of China (31601595, 31772113), the China Postdoctoral Science Foundation (2016M592844), the Shaanxi Postdoctoral Science Foundation (2016BSHEDZZ115), and the China Agriculture Research System (CARS-27).

Keywords fungal genome, Glomerella spp. 
conidia at a final concentration of $1 \times 10^{5}$ per milliliter. Total genomic DNA was extracted with a cetyltrimethylammonium bromide (CTAB)-based approach and were further gel-purified with the PippinHT DNA size selection system (Sage Science, Beverly, MA, U.S.A.) prior to nanopore sequencing with the PromethION platform (Oxford Nanopore, Oxford). Both genomic DNA extraction and nanopore sequencing were performed at Nextomics Biosciences Sequencing Center, Wuhan, China. A total of 357,984 reads (total bases $7.56 \mathrm{~Gb}, \mathrm{~N}_{50}=$ $27,858 \mathrm{bp}$ ) were obtained, from which draft assemblies were generated using both Canu v. 1.8 (Koren et al. 2017) and Flye v. 2.4.2 (Kolmogorov et al. 2019), using default parameters and an estimated genome size of $60 \mathrm{Mb}$ (Liang et al. 2018a). Both assemblies were highly contiguous (21 and 27 contigs, respectively), whereas more contigs from the Flye assembly were capped with copies of the telomeric repeat (TTAGGG). This Flye assembly was thus chosen for downstream refinements. The Flye assembly was polished with Pilon v. 1.2.3 (Walker et al. 2014), using previously generated Illumina reads (5.8 Gb 100-bp pair-ended reads [Liang et al. $2018 \mathrm{a}])$, resulting in changes at $231,173(0.39 \%)$ positions. To further refine the genome, we performed $\mathrm{Hi}-\mathrm{C}$ sequencing using $\mathrm{PDB}-$ derived young mycelia as the source material for genomic DNA. Library generation and DNA sequencing were performed at Annoroad Genome Sequencing Center (Beijing). Restriction with Mbol was used to fragment the genomic DNA and the generated library was sequenced with the HiSeq2500 platform. The 150-bp pair-end reads (filtered, 77.3 M) were mapped against the Pilon-polished Flye assembly, using HiC-Pro v. 2.11.1 (Servant et al. 2015). For simplicity, 12 short contigs (three shorter than $1 \mathrm{~kb}$, five between 1 and $6 \mathrm{~kb}$, four between 29.6 and $55.5 \mathrm{~kb}$ ) were omitted from the $\mathrm{Hi}-\mathrm{C}$ analysis. Two of these short contigs represented the mitochondrial genome, while nine others were repetitive sequences based on BLASTN search against the genome assembly. After Hi-C read mapping, 30.6 M (47.3\%) nonredundant valid interactions were obtained. The fractions of trans-interaction, cis short-range interaction and cis long-range interaction were 29,23 , and $48 \%$, respectively, which were in accordance with a high quality $\mathrm{Hi}-\mathrm{C}$ library (Servant et al. 2015). These $\mathrm{Hi}-\mathrm{C}$ interaction data were used to guide the assembly refinement by running SALSA (Ghurye et al. 2017) and by manual examination aided by Juicebox v. 1.9.8 (Durand et al. 2016). Two contigs were joined in an end-to-end manner, and two regions showing complex interaction patterns were cut from the original contigs and were treated as independent scaffolds.

The final $\mathrm{Hi}-\mathrm{C}$ validated 1104-7 assembly had a total size of $58.69 \mathrm{Mb}$, an overall GC content of $53.11 \%$, and was comprised of 16 scaffolds (S1 to S16) with sizes ranging from $165 \mathrm{~kb}$ to $7.12 \mathrm{Mb}$. Three scaffolds contained telomeric repeats on both ends and nine scaffolds contained telomeric repeats on one end. The $3^{\prime}$ end of scaffold 3 (S3) corresponded to ribosomal DNA repeats. In the Hi-C contact map, the strongest interaction signal was diagonal, supporting the integrity of the assembly. In addition, based on the Hi-C interaction signal pattern, putative centromeres could be identified from 12 scaffolds ( $S 1$ to $S 10, S 12$, S13), all of which contained extremely AT-rich DNA. The assembly completeness was evaluated by searching for conserved genes in the fungi_odb9 library (1,438 total genes), using BUSCO v1.2 (Simão et al. 2015). From this analysis 1,404 had hits as 'complete' genes and 1,311 had hits as 'complete and single-copy' genes. Both these statistics were higher than those for the previous Illumina assembly (1,392 and 1,301, respectively). Moreover, 34 fungal core genes had fragmented hits or lacked a hit in the nanopore assembly using Flye, whereas 46 genes were in this category in the Illumina assembly.

To predict gene structures, GeneMark-ET (Lomsadze et al. 2014) was trained with mapped RNA-seq reads derived from representative biological samples (conidia, cellophane-induced appressoria, cellophane infectious hyphae, developing perithecia, and infected apple leaves). Perithecial samples were collected from a 'mating' line formed between 1104-7 and its sibling strain 1104-6 on oatmeal agar. Procedures to collect samples for other cell types have been described previously (Liang et al. 2018b). Augustus v. 3.1 (Stanke et al. 2006) was trained with gene models of the Joint Genome Institute (JGI) Glomerella cingulata 23 strain (from the JGI MycoCosm database). G. cingulata was the name previously used for the sexual stage of $C$. fructicola and other members of the $C$. gloeosporioides species complex clade. RECON v. 1.08 (Bao and Eddy 2002) and RepeatScout v. 1.0 .5 (Price et al. 2005) were used for the de novo identification of repetitive sequences. Finally, integrative gene prediction was performed with Maker2 v. 2.31.8 (Holt and Yandell 2011), using input files including the generated GeneMark-ET $\mathrm{hmm}$ model file, Augustus hmm model file, de novo assembled transcripts, repeat consensus fasta file, and predicted proteins of the G. cingulata 23 strain. De novo assembled transcripts were obtained with Stringtie v. 1.3.6 (Pertea et al. 2015) and Transdecoder v. 5.4.0.

Vol. 33, No. 6, 2020 / 791 
Table 1. Comparison of the assembly statistics of Colletotrichum fructicola isolate 1104-7 (new nanopore assembly and previous Illumina assembly) and isolate Nara gc5

$\begin{array}{lccc}\text { Features } & \text { Nara gc5 } & \mathbf{1 1 0 4 - 7} \text { (IIlumina) } & \mathbf{1 1 0 4 - 7} \text { (nanopore) } \\ \text { Total length }(\mathrm{Mb}) & 55.61 & 57.08 & 58.69 \\ \text { Scaffold number } & 1,241 & 684 & 16 \\ \mathrm{~N}_{50}(\mathrm{Mb}) & 0.11 & 0.34 & 4.99 \\ \mathrm{~L}_{50} & 152 & 50 & 5 \\ \text { Putative centromeres } & \text { Not determined } & \text { Not determined } & 12 \\ \text { BUSCO complete }(\%) & 94.58 & 96.80 & 97.64 \\ \text { Genes }^{\text {a }} & 15,381 & 17,827 & 18,121 \\ \text { Secondary metabolism key enzymes }_{\text {Cytochrome P450s }} & 83 & 88 & 88 \\ \text { Carbohydrate-active enzymes } & 272 & 276 & 283 \\ \text { Proteases } & 1,112 & 1,211 & 617 \\ \text { Transcription factors } & 593 & 617 & 607 \\ \text { Transporters } & 432 & 586 & 615 \\ \text { Secreted proteins } & 1,067 & 1,109 & 1,106 \\ \text { Candidate effectors } & 1,225 & 1,440 & 3,470 \\ \text { a } & 292 & 372 & 378\end{array}$

a Predictions were based on a previously described pipeline (Liang et al. 2018a), except for the candidate effectors, which were predicted based on EffectorP 2.0 (Sperschneider et al. 2018) with default parameters.

The integrated pipeline predicted 18,121 protein-coding genes, similar to the number predicted in the previous Illumina assembly annotation $(17,827)$. BLASTP search (E-value cutoff $=1 \mathrm{e}-05)$ revealed that the new annotation included 1,850 (10.2\%) new genes lacking a match in the previous annotation, whereas the old annotation included 1,116 (6.27\%) unique genes. Among the genes that were unique to the Illumina annotation, $902(80.8 \%)$ lacked a significant (E-value cutoff $=1 \mathrm{e}-05)$ BLASTP hit in the nonredundant National Center for Biotechnology Information (NCBI) protein database, and 447 (40.1\%) lacked any RNA-seq reads mapping from three in-vitro samples and one in-planta sample. Conversely, among genes newly identified from annotation of the nanopore Flye assembly, 1,462 (79.0\%) lacked a significant BLASTP hit and 311 (16.81\%) lacked RNA-seq mapped reads. Based on the new gene annotation, we re-predicted virulence-related genes including secondary metabolite key enzymes, cytochrome P450s, carbohydrate-active enzymes, proteases, transcription factors, transporters, small secreted proteins, and candidate effectors (Table 1). Gene numbers in each functional category were similar between the two gene annotation versions. Among sequenced Colletotrichum genomes, $C$. fructicola and $C$. gloeosporioides encoded the largest repertoire of putative transporters and cytochrome P450s (unpublished data).

The long-read sequencing-based assembly of $C$. fructicola 1104-7 is by far the most complete genome assembly of $C$. fructicola and serves as an important resource for further genomic studies with this important phytopathogen. The assembled genome has been deposited at GenBank under the accession number MVNS00000000, and the gene annotations have been deposited in figshare. Nanopore and Hi-C Illumina data are available in the NCBI Sequenced Read Archive under accession numbers SRR10423968 and SRR10416202, respectively.

\section{Author-Recommended Internet Resources}

figshare: https://figshare.com/s/76d0c0bc1e53fc9ł3a5e

JGI MycoCosm database: https://genome.jgi.doe.gov/programs/fungi/index.jsf

\section{Literature Cited}

Bao, Z., and Eddy, S. R. 2002. Automated de novo identification of repeat sequence families in sequenced genomes. Genome Res. 12:1269-1276.

Bragança, C. A. D., Silva, L. L., Haddad, F., and Oliveira, S. A. S. 2016. First report of Colletotrichum fructicola causing anthracnose in cassava (Manihot esculenta) in Brazil. Plant Dis. 100:857.

Durand, N. C., Robinson, J. T., Shamim, M. S., Machol, I., Mesirov, J. P., Lander, E. S., and Aiden, E. L. 2016. Juicebox provides a visualization system for $\mathrm{Hi}-\mathrm{C}$ contact maps with unlimited zoom. Cell Syst. 3:99-101.
Gan, P., Ikeda, K., Irieda, H., Narusaka, M., O'Connell, R. J., Narusaka, Y., Takano, Y., Kubo, Y., and Shirasu, K. 2013. Comparative genomic and transcriptomic analyses reveal the hemibiotrophic stage shift of Colletotrichum fungi. New Phytol. 197:1236-1249.

Ghurye, J., Pop, M., Koren, S., Bickhart, D., and Chin, C.-S. 2017. Scaffolding of long read assemblies using long range contact information. BMC Genomics 18:527.

Holt, C., and Yandell, M. 2011. MAKER2: An annotation pipeline and genomedatabase management tool for second-generation genome projects. BMC Bioinformatics 12:491. 
Hu, W. L., Ma, Y. Z., and Chen, J. Z. 2019. First report of Citrus sinensis anthracnose caused by Colletotrichum fructicola in China. Plant Dis. 103:1018.

Kolmogorov, M., Yuan, J., Lin, Y., and Pevzner, P. A. 2019. Assembly of long, errorprone reads using repeat graphs. Nat. Biotechnol. 37:540-546.

Koren, S., Walenz, B. P., Berlin, K., Miller, J. R., Bergman, N. H., and Phillippy, A. M. 2017. Canu: Scalable and accurate long-read assembly via adaptive $k$-mer weighting and repeat separation. Genome Res. 27:722-736.

Li, H., Zhou, G.-Y., Liu, J.-A., and Xu, J. 2016. Population genetic analyses of the fungal pathogen Colletotrichum fructicola on tea-oil trees in China. PLoS One 11: e0156841.

Liang, X., Shang, S., Dong, Q., Wang, B., Zhang, R., Gleason, M. L., and Sun, G. 2018b. Transcriptomic analysis reveals candidate genes regulating development and host interactions of Colletotrichum fructicola. BMC Genomics 19:557.

Liang, X., Wang, B., Dong, Q., Li, L., Rollins, J. A., Zhang, R., and Sun, G. 2018a. Pathogenic adaptations of Colletotrichum fungi revealed by genome wide gene family evolutionary analyses. PLoS One 13:e0196303.

Lomsadze, A., Burns, P. D., and Borodovsky, M. 2014. Integration of mapped RNASeq reads into automatic training of eukaryotic gene finding algorithm. Nucleic Acids Res. 42:e119.

Pertea, M., Pertea, G. M., Antonescu, C. M., Chang, T.-C., Mendell, J. T., and Salzberg, S. L. 2015. StringTie enables improved reconstruction of a transcriptome from RNA-seq reads. Nat. Biotechnol. 33:290-295.

Price, A. L., Jones, N. C., and Pevzner, P. A. 2005. De novo identification of repeat families in large genomes. Bioinformatics 21 (Suppl 1):i351-i358.

Prihastuti, H., Cai, L., Chen, H., McKenzie, E., and Hyde, K. 2009. Characterization of Colletotrichum species associated with coffee berries in northern Thailand. Fungal Divers. 39:89.
Rockenbach, M. F., Velho, A. C., Gonçalves, A. E., Mondino, P. E., Alaniz, S. M., and Stadnik, M. J. 2016. Genetic structure of Colletotrichum fructicola associated to apple bitter rot and Glomerella leaf spot in southern Brazil and Uruguay. Phytopathology 106:774-781.

Servant, N., Varoquaux, N., Lajoie, B. R., Viara, E., Chen, C.-J., Vert, J.-P., Heard, E., Dekker, J., and Barillot, E. 2015. HiC-Pro: An optimized and flexible pipeline for $\mathrm{Hi}-\mathrm{C}$ data processing. Genome Biol. 16:259.

Silva-Cabral, J. R. A., da Silva, J. L., Soares, L. S. H., Costa, J. F. O., Amorim, E. P. R., Lima, G. S. A., and Assunção, I. P. 2019. First report of Colletotrichum fructicola and $C$. tropicale causing anthracnose on orchids in Brazil. Plant Dis. 103:2672.

Simão, F. A., Waterhouse, R. M., loannidis, P., Kriventseva, E. V., and Zdobnov, E. M. 2015. BUSCO: Assessing genome assembly and annotation completeness with single-copy orthologs. Bioinformatics 31:3210-3212.

Sperschneider, J., Dodds, P. N., Gardiner, D. M., Singh, K. B., and Taylor, J. M. 2018. Improved prediction of fungal effector proteins from secretomes with EffectorP 2.0. Mol. Plant Pathol. 19:2094-2110.

Stanke, M., Keller, O., Gunduz, I., Hayes, A., Waack, S., and Morgenstern, B. 2006. AUGUSTUS: Ab initio prediction of alternative transcripts. Nucleic Acids Res. 34 (Web Server):W435-W439.

Walker, B. J., Abeel, T., Shea, T., Priest, M., Abouelliel, A., Sakthikumar, S., Cuomo, C. A., Zeng, Q., Wortman, J., Young, S. K., and Earl, A. M. 2014. Pilon: An integrated tool for comprehensive microbial variant detection and genome assembly improvement. PLoS One 9:e112963.

Wang, H. C., Huang, Y. F., Chen, Q., Wang, M. S., Xia, H. Q., Shang, S. H., and Zhang, C. Q. 2016. Anthracnose caused by Colletotrichum fructicola on tobacco (Nicotiana tabacum) in China. Plant Dis. 100:1235. 